\title{
Particle hydrogels based on hyaluronic acid building blocks
}

Elias Sideris, Donald R. Griffin, Yichen Ding, Shuoran Li, Westbrook Weaver, Dino Di Carlo Tzung Hsiai, Tatiana Segura*

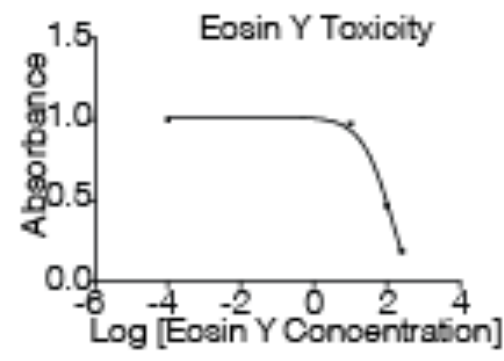

Supplemental Figure 1: MTS toxicity test of Eosin Y on human dermal fibroblasts

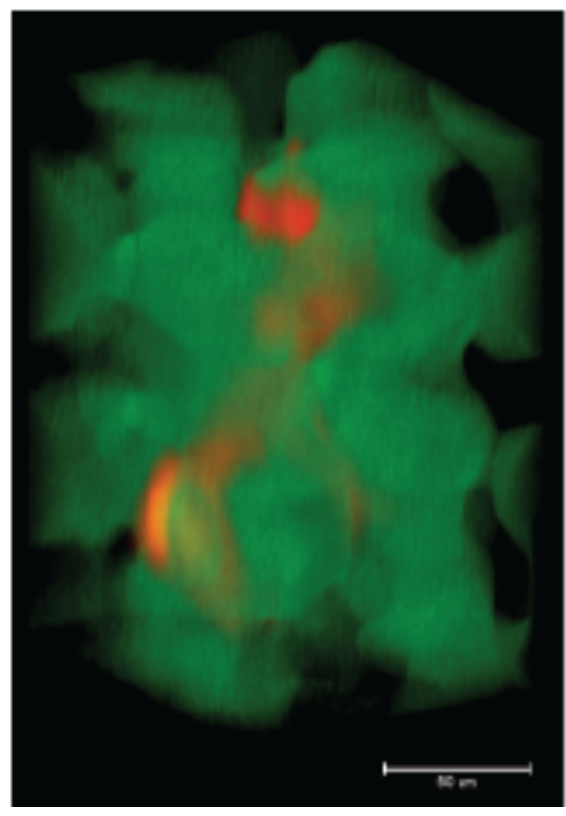

Supplemental Figure 2: Sheet confocal image of cells (red) spread in small pores (scale bar: $100 \mu \mathrm{m})$ formed by the annealed $\mu$ gels (green). 


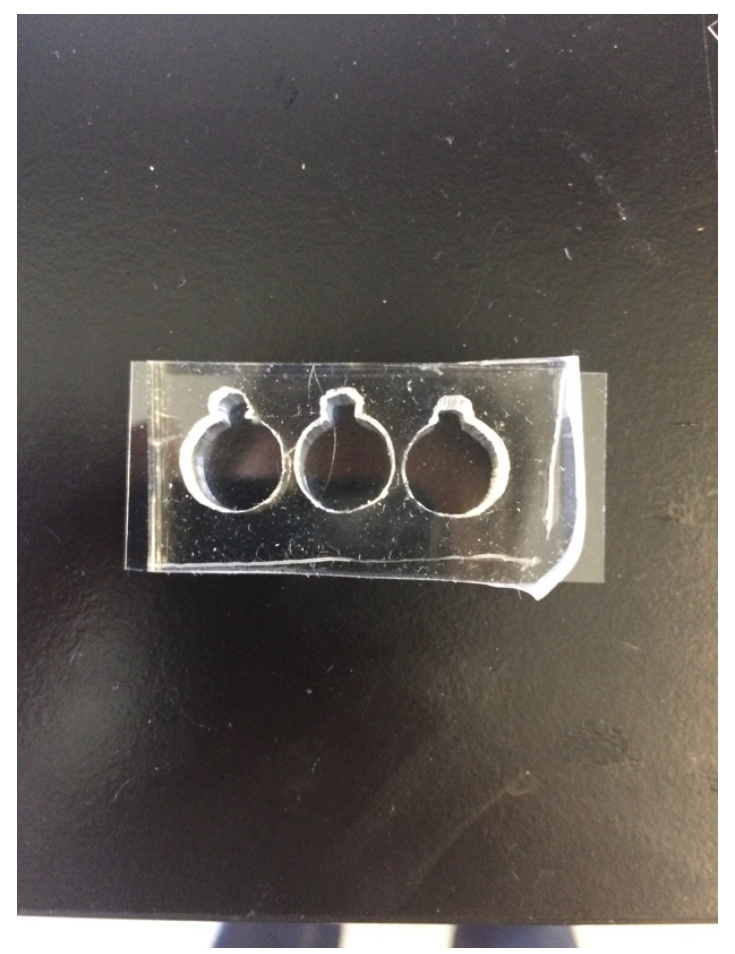

Supplemental Figure 3: PDMS molds used for culture of cells in MAP gel.

\section{Collagenase I Degradation}

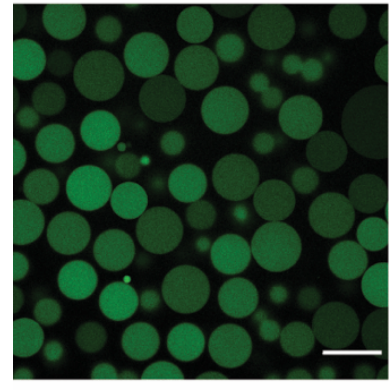

$\mathrm{t}=0 \mathrm{~min}$

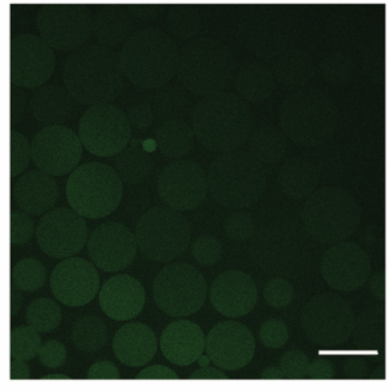

$\mathrm{t}=20 \mathrm{~min}$

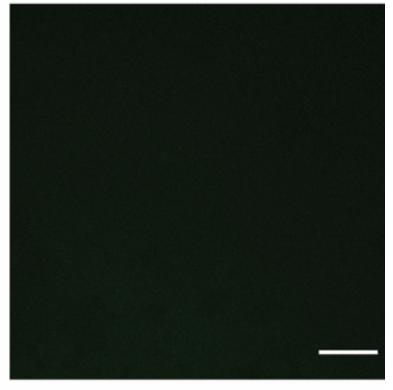

$\mathrm{t}=40 \mathrm{~min}$

Supplemental Figure 4: Collagenase I degradation: HA microgels were mixed in a solution of 20 $\mathrm{u} / \mathrm{ml}$ Collagenase I at room temperature and continuously imaged at the same location. Scale bar $=100 \mu \mathrm{m}$. 